\title{
LASER STOKES-POLARIMETRY OF THE ERYTHROCYTES SUSPENSION AT COMORBID COURSE OF CORONARY ARTERY DISEASE, DIABETES MELLITUS TYPE 2 AND ANEMIA
}

\author{
Nataliia Pavlyukovich, ${ }^{1}$ Oleksandr Pavlyukovich, ${ }^{2}$ Marta Garazdiuk, ${ }^{3}$ \\ Oleg Wanchuliak, ${ }^{4}$ Oleksandr Garazdiuk ${ }^{5}$
}

\begin{abstract}
:
Introduction: Investigation of the rheological properties of the erythrocytes is one of the crucial issues in the pathogenesis of most diseases of internal organs. Changes in the morphological structure of the red blood cell membrane serve as an early diagnostic criterion of coronary artery disease, diabetes mellitus type 2 and anaemia, thus a search for modern methods of investigation which can be used for early detection of erythrocytes membrane disorders is of great importance. Methods of laser polarimetry of the erythrocytes suspension smear are crucial in this area.

Objectives: Investigation of the possible structural changes of the erythrocytes membranes in patients with coronary artery disease, diabetes mellitus type 2 and anaemia of different degrees of severity.

Methods: For the objective assessment of the structural state of erythrocytes membrane laser polarimetry of the red cell suspension smear was applied. Set of the statistical points of the $1^{\text {st }}-4^{\text {th }}$ grades was calculated and their effectiveness in the early pre-clinical detection of the erythrocytes membrane disorder was established.

Results: Images of the erythrocytes suspension samples, registered in the co-axial and crossed planes of the polarizer and analyser transmissions, regardless of the investigated group, are coordinating heterogeneous. However, obvious signs of the changes of the images structure depending on the presence and type of pathology are not visually detected. As the basis of an objective approach, a statistical analysis with histograms of the coordinate distributions of the random variables and a statistical calculation of the statistical points of the $1^{\text {st }}-4^{\text {th }}$ grades should be used. All statistical points, which describe the coordinate distributions of the various parameters of the Stokes vector, have individual sets of values and may be used for the differential diagnosis of the coronary artery disease, diabetes mellitus type 2 and anaemia.

Conclusions: Interconnections between the values of the sets of the statistical points of the $1^{\text {st }}-4^{\text {th }}$ grades, which characterized coordinate distributions of the intensity, azimuth and ellipticity of the polarization of the erythrocytes suspension laser images of the elderly and senile patients with comorbidity were established by means of laser polarimetry. The most sensitive were asymmetry and kurtosis, which can be used for early detection of the erythrocytes membrane disorder when routine methods of its visualization and estimation still are not able to be applied.
\end{abstract}

UDC Classification: 616.1; DOI: http://dx.doi.org/10.12955/cbup.v5.1059

Keywords: coronary artery disease, diabetes mellitus, anemia, laser, polarimetry

\section{Introduction}

Laser radiation during interaction with biological tissue (BT) can be absorbed and dissipated. Ushenko et al. (2007) found that each of these processes has some information on the micro- and macrostructure of the biological environment. According to Ushenko and Bachynskiy (2007), modelling the structure of the BT is based upon the idea that it is a two-component structure that consists of:

- optical-anisotropic component - the matrix predominantly formed by the fibrous tissue components (collagen fibers, proteins, fibrin fibers, etc.). This component is able to change the main parameters of the laser radiation during its passage through the layer of BT;

- amorphous component - the components of BT, which has no fibrous structure. The latter is optically neutral, i.e. one that does not change the basic characteristics of the beam of laser radiation while passing through $\mathrm{BT}$.

The possibility of usage of the laser polarimetry methods for objective assessment of the erythrocytes' membranes is caused by the presence in their architecture the significant part of the specific protein structures which, in its turn, are anisotropic from the optical point of view, that is, they are able to change the properties of the laser radiation during its passage through BT. Protein components of the

\footnotetext{
${ }^{1}$ Bukovinian State Medical University, Ukraine, natasha.pavlyukovich@gmail.com

${ }^{2}$ Bukovinian State Medical University, Ukraine, olexandr.pavlyukovich@gmail.com

${ }^{3}$ Bukovinian State Medical University, Ukraine, m.garazdiuk@gmail.com

${ }^{4}$ Bukovinian State Medical University, Ukraine, sudmed@bsmu.edu.ua

${ }_{5}^{5}$ Bukovinian State Medical University, Ukraine, olexandr.harazdiuk@gmail.com
} 
erythrocyte membrane, unlike lipid, have the clear complicated hierarchical structure due to their complex level of organization.

It is known by Korin, et al., (2007) and Steiner et al., (2009) that spectrin of the erythrocyte membrane has a penta- or hexagonal structure, which is formed by the tetramers of its molecules, that are linked to short actin microfilaments on both ends. It was shown by Krylov et al., (2010) that spectrin cytoskeleton of the erythrocyte maintains the definite form of the cell, therefore structural change in the ordering of the molecules can serve as a prerequisite change of the morphological structure of the erythrocyte membrane and the resulting disorder of its functional properties.

Aim: to investigate possible structural changes of the erythrocytes membranes in patients with coronary artery disease (CAD), diabetes mellitus (DM) type 2 and anaemia of different degrees of severity by means of laser polarimetry methods.

Material and Methods of Investigation. 88 patients with CAD, DM type 2 and anaemia, hospitalized to the cardiological department of the Chernivtsi Regional Hospital for War Veterans, were under investigation. They were randomized into subgroups according to the comorbid pathology: I - CAD patients with concomitant DM type 2 ( $\mathrm{n}=12)$, II -patients with CAD, complicated by concomitant anaemia and DM type $2(\mathrm{n}=76)$. The control group for comparative studies comprised 12 patients with CAD without comorbid pathology.

For the objective assessment of the structural state of erythrocytes membrane, laser polarimetry of the red cell suspension smear was applied. Scheme of the optical laser polarimeter is presented in Figure 1.

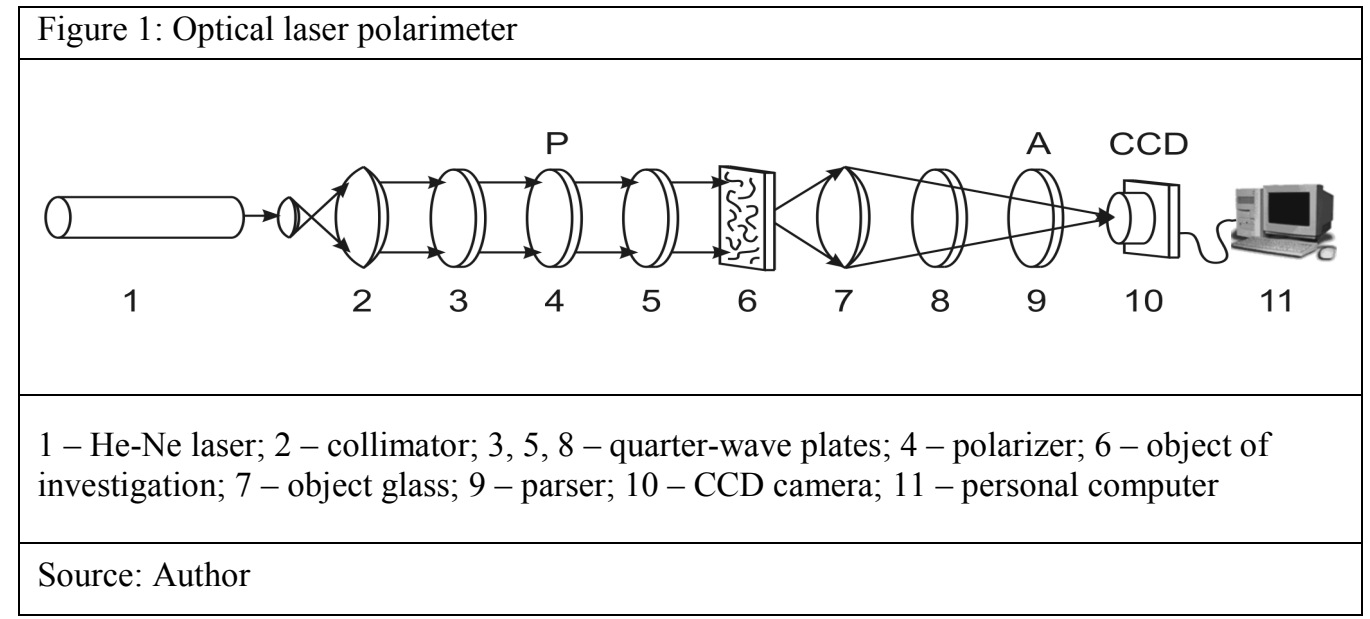

Irradiation was conducted by the beam $(\varnothing=104 \mathrm{mkm})$ of the He-Ne laser (1) with a wavelength $\lambda=0,6328 \mathrm{~mm}$. With the help of the polarizing film (quarter-wave plate and polarizer) different states of polarization of the illuminating beam were formed. Polarization images of the layers of the erythrocyte suspension (6) were formed in the plane of the light-sensitive pad (800x600) of the CCD camera (10) through the object glass (7), the resolution of which was sufficient for the measurements in the size range of structural laser images of the erythrocytes suspension (2-2000 microns).

To assess coordinate distributions of the random variables their histograms were used; after that we calculated a set of the statistical points of the $1^{\text {st }}-4^{\text {th }}$ grades.

Results of the Investigation. To determine the main 2D-parameters of the Stokes vectors distribution in the plane of the laser image of the erythrocyte suspension we determined the value of the Stokes vector of such images by means of six measurements of the intensity in case of the following polarization filtering conditions using a polarizer-analyzer.

The plane of the polarizer-analyzer transmission on the angle $\Theta=0^{\circ}$ was oriented and intensity distribution $I_{0}(m \times n)$ of the laser images was measured (Figure 2). Then the plane of the polarizeranalyzer transmission on the angle $\Theta=90^{\circ}$ was changed and coordinate intensity distribution $I_{90}(m \times n)$ of the laser images was detected (Figure 3). Based on the definition of the Stokes vector $\mathrm{S}$ we established its first parameter $S_{I}$ which characterized the full intensity $I$ according to the following ratio $-S_{I}=I_{0}+I_{90}$ (Figure 4). 

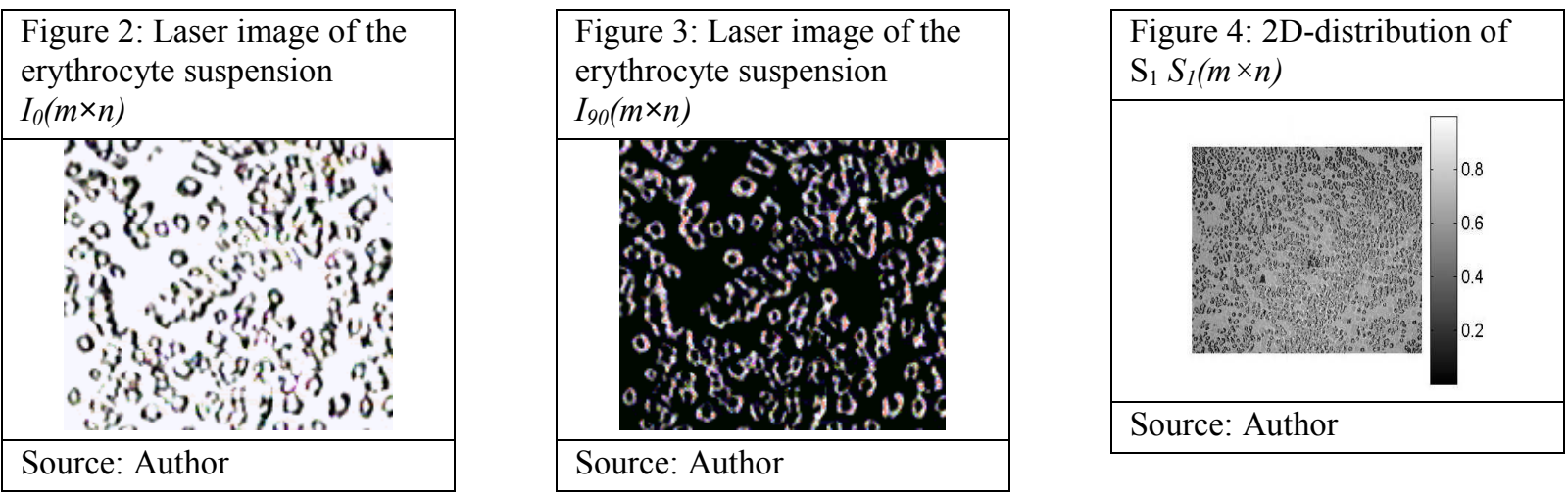

After that the plane of the polarizer-analyzer was oriented on the angle and coordinate distribution of $I_{45}(m \times n)$ of the erythrocytes suspensions laser images was measured (Figure 5). Later on, the plane of the polarizer-analyzer transmission on the angle was changed and coordinate intensity distribution $I_{135}(m \times n)$ of the red blood cells polarization images was measured (Figure 6). According to the ratio $S_{3}$ $=I_{45}-I_{135}$ the $S_{3}$ Stokes parameter was found which characterized the azimuth distribution of the laser images polarization (Figure 7).

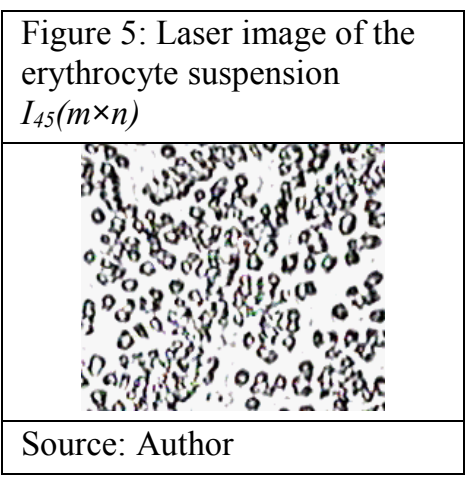
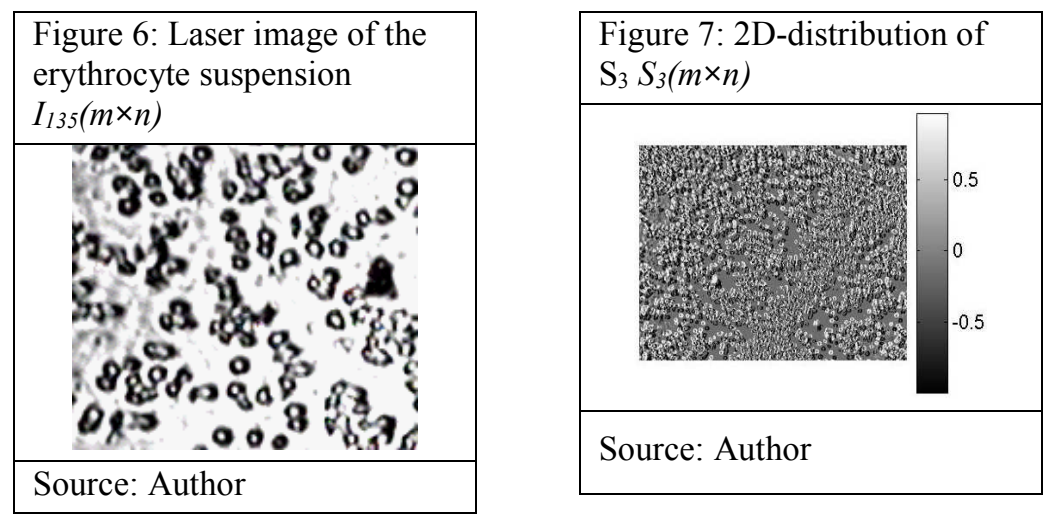

To measure the fourth parameter of the Stokes vector $S_{4}$ quarter-wave-length plate was installed in the way of the laser beam, so that the axis of its maximum speed was oriented at the angle of $0^{\circ}$ to the plane of the probe laser beam polarization. The plane of the polarizer-analyzer transmission was oriented on the angle and the coordinate intensity distribution of the right-circulating polarized light was measured (Figure 8).

\begin{tabular}{|l|}
\hline Figure 8: Laser image of the \\
erythrocyte suspension \\
$I_{0}(m \times n)$ in the right- \\
circulating light \\
\hline \\
Source: Author \\
\hline
\end{tabular}
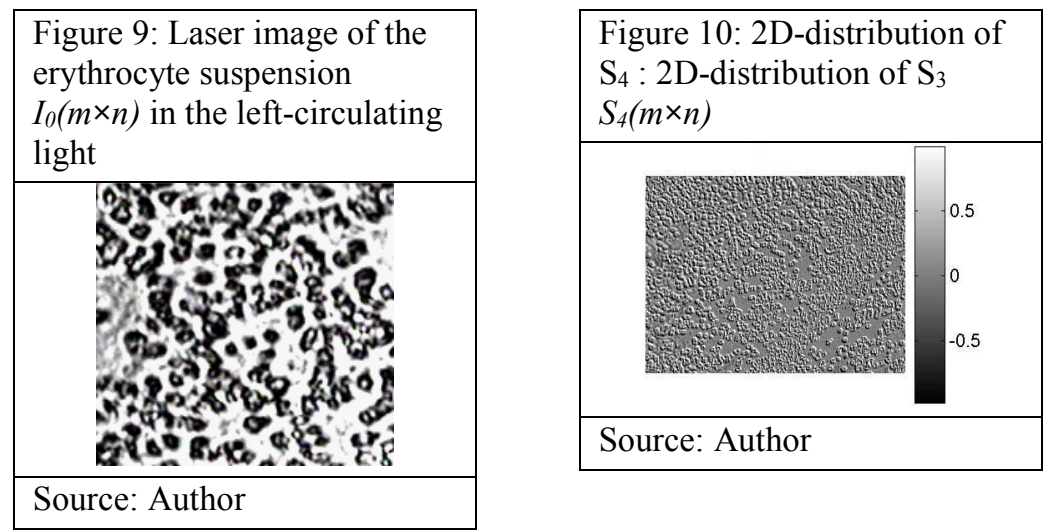

Finally, we oriented the plane of the polarizer-analyzer transmission on the axis of the maximum speed of the quarter-wave-length plate on the angle and measured the intensity distribution of the leftcirculating polarized light (Figure 9). According to the ratio coordinate distribution of the fourth parameter of the Stokes vector $S_{4}$ of the red cell suspension laser image was calculated (Figure 10), which described the birefringence of the biological layer of the erythrocytes suspension. 
Having analyzed the images of the erythrocytes suspension samples, registered in the co-axial $\left(\Theta=0^{\circ}\right)$ and crossed $\left(\Theta=90^{\circ}\right)$ planes of the polarizer and analyzer transmissions, we established that all of them, regardless of the investigated group, are coordinating heterogeneous (Figure 11).

Figure 11: Images of the erythrocytes suspension samples of the investigated patients

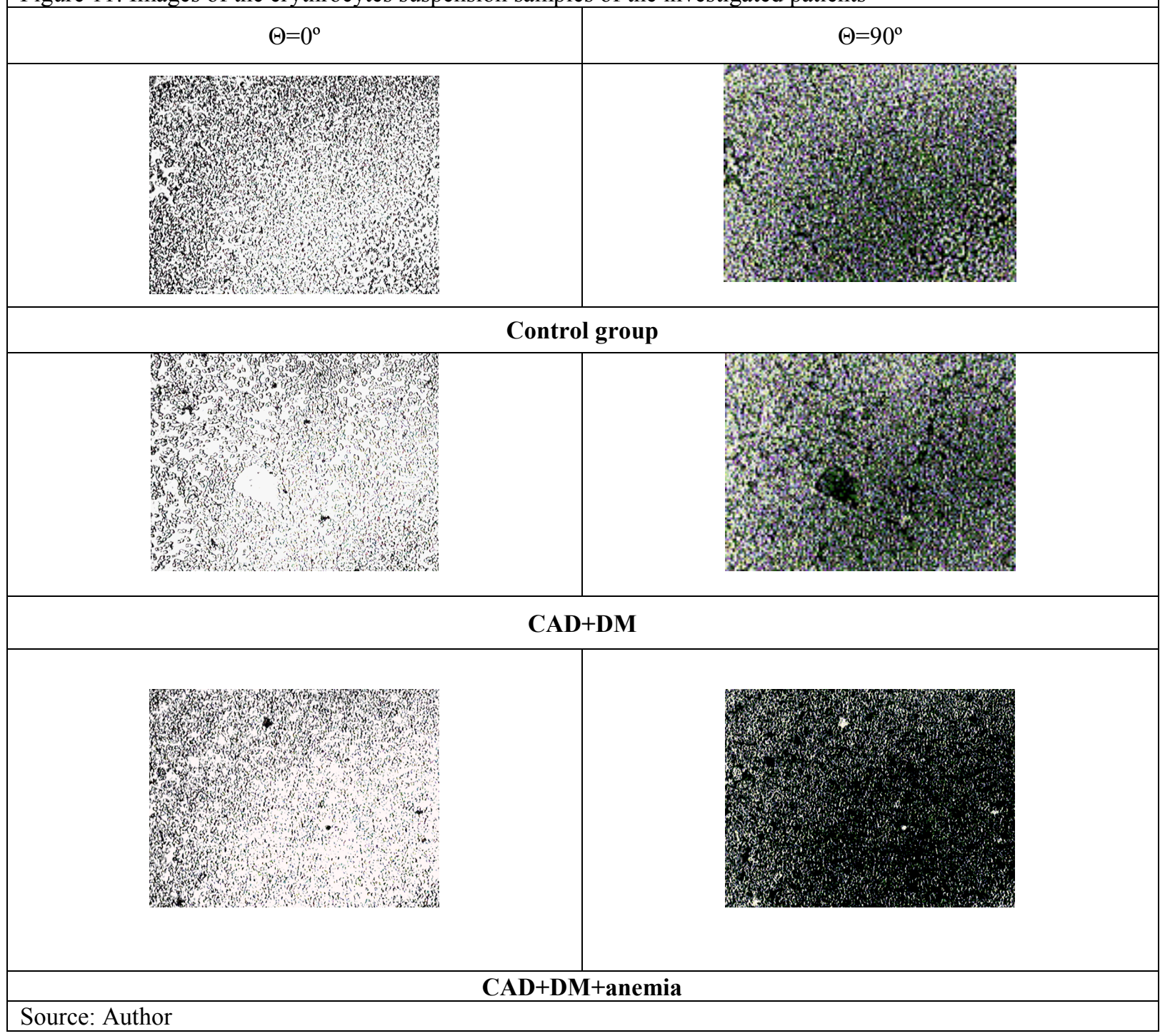

Red blood cells contained optically anisotropic areas of the substance, which were visualized in the form of the enlighten areas in the crossed polarizer and analyzer $\left(\Theta=90^{\circ}\right)$. However, obvious signs of the changes of the images structure depending on the presence and type of pathology were not visually detected.

According to the above-mentioned facts we aimed at the determining of the objective (quantitative) criteria for the differentiation of the patterns of the polarization-inhomogeneous laser images and search for possible connections between the changes in the red blood cells structure and investigated comorbidity.

As the basis of such objective approach statistical analysis with histograms of the coordinate distributions of the random variables and statistical calculation of the statistical points of the $1^{\text {st }}-4^{\text {th }}$ grades was used. Figure 12 shows the coordinate $(1,3,5)$ and probability $(2,4,6)$ distribution of the values of the parameters of the Stokes vector $S_{i=1 ; 3 ; 4}(m \times n)$ at the points of the erythrocyte suspensions laser images of the patients of the control group. The obtained results clearly visibly represent the coordinate heterogeneous and individual types of the histograms of the distribution of the values of intensity $\left(S_{1}(m \times n)\right)$, azimuth $\left(S_{3}(m \times n)\right)$ and ellipticity $\left(S_{4}(m \times n)\right)$ of the polarization of the erythrocyte suspensions laser images of the patients of the control group. 


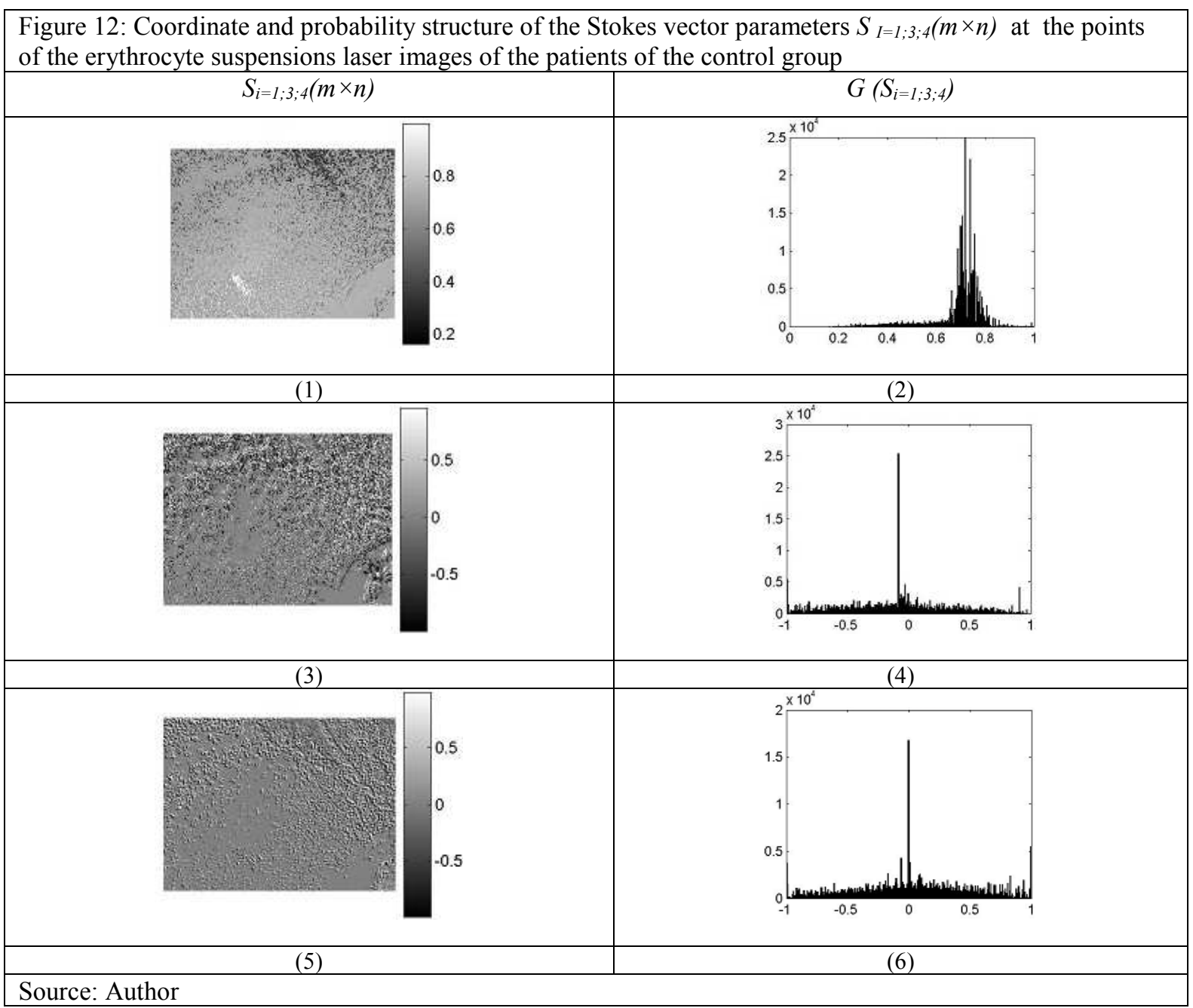

To have the quantitative criteria to characterize such complex images, we calculated a set of statistical points of the $1^{\text {st }}-4^{\text {th }}$ grades in the statistically significant groups according to the known algorithms. Average values of the obtained parameters and ranges of their difference within the control group are given in the Table.

\begin{tabular}{|c|c|c|c|c|}
\hline & Statistical point & Control (n=12) & CAD+DM (n=12) & CAD+DM+anemia $(n=76)$ \\
\hline \multirow{4}{*}{$S_{I}$} & $\begin{array}{c}\boldsymbol{M} \\
\text { medium }\end{array}$ & $0,67 \pm 0,072$ & $0,69 \pm 0,017$ & $0,69 \pm 0,019$ \\
\hline & $\begin{array}{c}\sigma \\
\text { variance }\end{array}$ & $0,15 \pm 0,012$ & $0,11 \pm 0,009$ & $0,095 \pm 0,012$ \\
\hline & $\begin{array}{c}A \\
\text { asymmetry }\end{array}$ & $0,32 \pm 0,021$ & $0,52 \pm 0,011$ & $2,42 \pm 0,191^{*}$ \\
\hline & $\begin{array}{c}\boldsymbol{E} \\
\text { kurtosis }\end{array}$ & $0,21 \pm 0,028$ & $0,44 \pm 0,053$ & $2,23 \pm 0,421^{*}$ \\
\hline \multirow{4}{*}{$S_{3}$} & $\begin{array}{c}\boldsymbol{M} \\
\text { medium }\end{array}$ & $0,01 \pm 0,003$ & $0,03 \pm 0,005$ & $0,03 \pm 0,004$ \\
\hline & $\begin{array}{c}\sigma \\
\text { variance }\end{array}$ & $0,09 \pm 0,015$ & $0,07 \pm 0,032$ & $0,07 \pm 0,028$ \\
\hline & $\begin{array}{c}\boldsymbol{A} \\
\text { asymmetry }\end{array}$ & $0,05 \pm 0,002$ & $0,065 \pm 0,001^{*}$ & $0,14 \pm 0,002 * \S$ \\
\hline & $\begin{array}{c}\boldsymbol{E} \\
\text { kurtosis }\end{array}$ & $3,21 \pm 0,11$ & $3,78 \pm 0,083$ & $3,86 \pm 0,310$ \\
\hline \multirow{4}{*}{$S_{4}$} & $\begin{array}{c}\boldsymbol{M} \\
\text { medium }\end{array}$ & $0,02 \pm 0,002$ & $0,025 \pm 0,0023$ & $0,07 \pm 0,002 * \S$ \\
\hline & $\begin{array}{c}\sigma \\
\text { variance }\end{array}$ & $0,26 \pm 0,037$ & $0,14 \pm 0,059$ & $0,12 \pm 0,076$ \\
\hline & $\begin{array}{c}A \\
\text { asymmetry }\end{array}$ & $0,07 \pm 0,009$ & $0,095 \pm 0,0080$ & $0,14 \pm 0,012^{*}$ \\
\hline & $\begin{array}{c}\boldsymbol{E} \\
\text { kurtosis }\end{array}$ & $2,140 \pm 0,33$ & $2,310 \pm 0,19$ & $4,52 \pm 0,25^{*}$ \\
\hline
\end{tabular}


Analysis of the results revealed that all the statistical points of the $1^{\text {st }}-4^{\text {th }}$ grades, which described the coordinate distributions of the various parameters of the Stokes vector, had individual sets of values. Thus, we found the evaluability of the statistical parameters to distinguish the intensity, azimuth and ellipticity of the polarization of the red cell suspension laser images.

Figure 13 demonstrates that coordinate distributions of the Stokes vector parameters $S_{i=1 ; 3 ; 4}(m \times n)$ of the polarization-inhomogeneous image of the erythrocyte suspensions layer of the patients with coronary artery disease and comorbid diabetes mellitus type 2 are different from the previously analysed (Figure 10) - the spread of the random values of the polarization azimuth $S_{i=3}(m \times n)$ and ellipticity $S_{i=4}(m \times n)$ is gradually decreasing. This results in the decreasing of the variance $\sigma$, and, conversely, increasing of the asymmetry $A$ and kurtosis $E$.

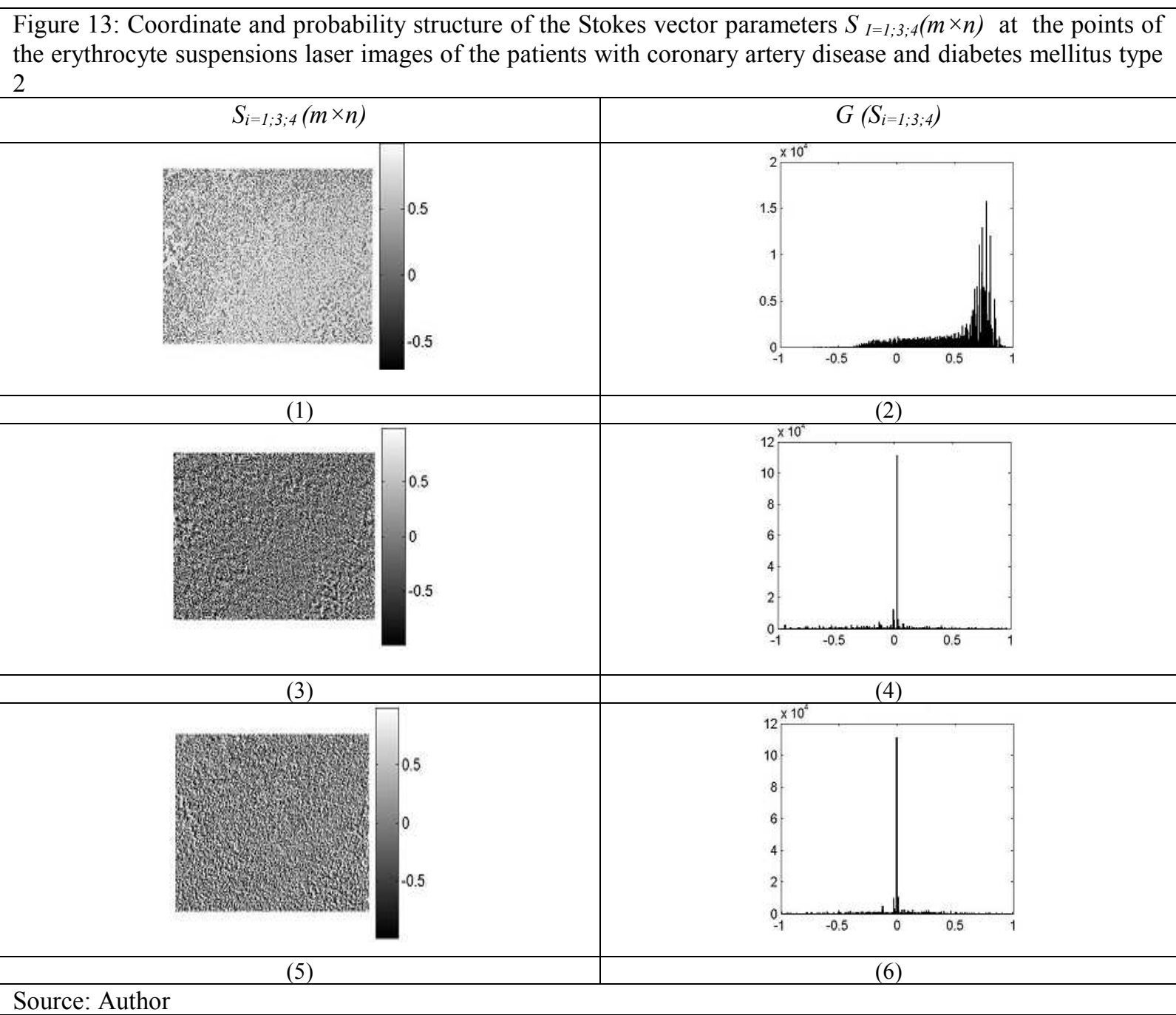

It was established that a set of the statistical points of the $1^{\text {st }}-4^{\text {th }}$ grades, which characterized the coordinate distribution of the Stokes vector parameters of the polarization-inhomogeneous images of the erythrocyte suspensions samples of the patients with coronary artery disease and diabetes mellitus type 2, the following differences compared with the same parameters of the samples of the control group are typical: medium $M\left(S_{i=1 ; 3 ; 4}\right)$ - approximately unchanged; variance $-\sigma\left(S_{i=1}\right)$ - decreased 1,36 times, $\sigma\left(S_{i=3}\right)-1,29$ times, $\sigma\left(S_{i=4}\right)-1,86$ times correspondently; asymmetry $-A\left(S_{i=1}\right)$ - increased 1,63 times, $A\left(S_{i=3}\right)-1,30$ times, $A\left(S_{i=4}\right)-1,36$ times correspondently; kurtosis $E\left(S_{i=1}\right)$ - increased 2,09 times, $E\left(S_{i=3}\right)-1,18$ times, $E\left(S_{i=4}\right)-1,08$ times correspondently.

The results of the laser polarimetry of the red blood cell suspension smears of the patients with CAD, DM type 2 and moderate anaemia are illustrated in Figure 14. It was found that the values of the statistical points of the $1^{\text {st }}-4^{\text {th }}$ grades, which characterized the coordinate distribution of the Stokes vector parameters of polarization-inhomogeneous images of the erythrocyte suspensions samples of 
the patients with $\mathrm{CAD}, \mathrm{DM}$ type 2 and moderate anaemia, differed statistically from the similar parameters of the samples of the control group (Table): medium $M\left(S_{i=1 ; 3 ; 4}\right)$ - approximately unchanged; variance $-\sigma\left(S_{i=1}\right)$ - decreased 1,58 times, $\sigma\left(S_{i=3}\right)-1,28$ times, $\sigma\left(S_{i=4}\right)-2,17$ times correspondently; asymmetry - $A\left(S_{i=1}\right)$ - increased 7,56 times, $A\left(S_{i=3}\right)-2,8$ times, $A\left(S_{i=4}\right)$ - y 2,0 times correspondently; kurtosis $-E\left(S_{i=1}\right)$ - increased 10,62 times, $E\left(S_{i=3}\right)-1,20$ times, $E\left(S_{i=4}\right)-2,11$ times correspondently.

Figure 14: Coordinate and probability structure of the Stokes vector parameters $S_{I=1: 3: 4}(m \times n)$ at the points of the erythrocyte suspensions laser images of the patients with coronary artery disease, diabetes mellitus and moderate anaemia

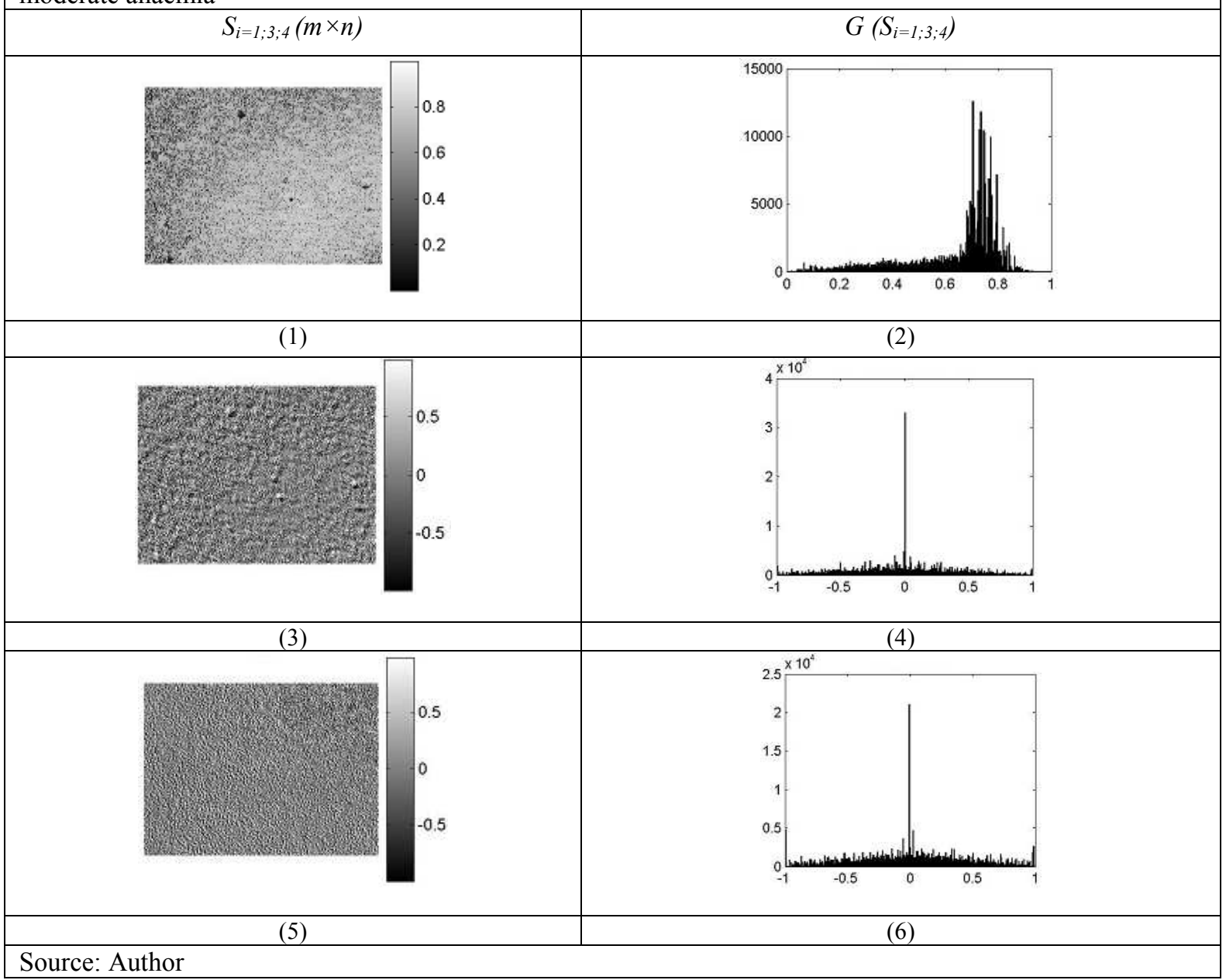

Conclusion. Thus, interconnections between the values of the sets of the statistical points of the $1^{\text {st }}-4^{\text {th }}$ grades $M ; \sigma ; A ; E$, which characterized coordinate distributions of the intensity $S_{l}(m \times n)$, azimuth $S_{3}(m \times n)$ and ellipticity $S_{4}(m \times n)$ of the polarization of the erythrocytes suspension laser images of the elderly and senile patients with comorbidity were established by means of laser polarimetry. Methods of the laser polarimetry of the red blood cells smear with the following analysis of the statistical points of the 1st - 4th grade might be used for early diagnosis of the structural and functional changes of the erythrocytes in patients with a combined course of coronary artery disease, diabetes mellitus type 2 and anaemic syndrome, especially at pre-clinic stages

\section{References}

Korin N., Bransky A. and Dinnar U. (2007) Theoretical model and experimental study of red blood cell (RBC) deformation in microchannels. Journal of Biomechanics. 40 (9), 2088-2095.

Krylov V.N., Deryugina A.V., Grishina A.A. (2010) Changes of the electrophoretic activity of the erythrocytes and lipid spectrum of their membranes at different stress impacts. Hematology and Transfusiology. 3, 40-44 (in Russian)

Steiner L.A., Maksimova Y., Schulz V. and Wong C. (2009) Molecular and cellular biology. 29 (20). 5399-5412.

Ushenko O.G. and Bachynskiy V.T. (2007) Laser nephelometry of the biological tissues. 300. (in Ukrainian)

Ushenko O.G. Pishak V.P., Angel's'kij O.V. and Ushenko Yu.O. (2007) Laser polarization morphology of the biological tissues: statistical and fractal approaches. 314. (in Ukrainian) 